TRANSACTIONS OF THE

AMERICAN MATHEMATICAL SOCIETY

Volume 365, Number 5, May 2013, Pages 2297-2316

S 0002-9947(2012)05593-0

Article electronically published on November 7, 2012

\title{
THE ZERO LEVEL SET FOR A CERTAIN WEAK SOLUTION, WITH APPLICATIONS TO THE BELLMAN EQUATIONS
}

\section{J. ANDERSSON AND H. MIKAYELYAN}

\begin{abstract}
We will prove a partial regularity result for the zero level set of weak solutions to

$$
\operatorname{div}(B \nabla u)=0
$$

where $B=B(u)=I+(A-I) \chi_{\{u<0\}}$, where $I$ is the identity matrix and the eigenvalues of $A$ are strictly positive and bounded.

We will apply this to describe the regularity of solutions to the Bellman equations.
\end{abstract}

\section{INTRODUCTION}

In this paper we will be studying the zero level set for weak solutions to elliptic equations $\operatorname{div}(B(x) \nabla u)=0$ where $B$ has constant coefficients in the sets $\Omega^{+}(u)=$ $\Omega^{+}=\{u>0\}$ and $\Omega^{-}(u)=\Omega^{-}=\{u<0\}$. For simplicity we will assume $B$ to be the identity in $\Omega^{+}$, and $B=A$ in $\Omega^{-}$, where $A$ is a constant coefficient matrix with strictly positive and bounded eigenvalues. In particular. we assume that $\lambda|\xi|^{2} \leq \xi \cdot A \cdot \xi \leq \Lambda|\xi|^{2}$ for some constants $0<\lambda<\Lambda<\infty$ and all vectors $\xi \in \mathbf{R}^{n}$.

Our main goal is to describe the regularity of the zero level set. We will think of this problem as a free boundary problem. Throughout this paper the zero level set will be referred to as the free boundary and denoted $F=F(u)=F_{u}$.

Similar free boundary problems have been studied before by many authors, most notably by L. Caffarelli whom in a series of articles (see [3], [5] and [4]) studied the $C^{1, \alpha}$ regularity of the free boundary for two phase free boundary problems of our type.

In particular, [4, [5] and [3] consider the regularity properties of $u \in C\left(B_{1}\right)$ and its zero level set where

$$
\begin{array}{ll}
\Delta u=0 & \text { in } \Omega^{+}(u) \text { and } \Omega^{-}(u), \\
\left|\nabla u^{+}\right|=G\left(\left|\nabla u^{-}\right|\right) & \text {on } \Gamma=\{u=0\},
\end{array}
$$

where the condition $\left|\nabla u^{+}\right|=G\left(\left|\nabla u^{-}\right|\right)$is understood in a viscosity sense (see section 6). But for smooth enough solutions it means that the normal derivative of $u^{+}$at a point $x^{0} \in \Gamma$ equals $G$ of the normal derivative of $u^{-}$at $x^{0}$. The standard model is that $G(t)=\sqrt{1+t^{2}}$.

Caffarelli's method, which we will follow closely, involves three steps. First, in [4, measure theoretic properties of the free boundary are investigated. In particular, it is shown that the set $\Omega^{+}(u)$ is a set of locally finite perimeter, which implies that the measure theoretic normal exists almost everywhere or, equivalently, the free, boundary is asymptotically flat almost everywhere. The second step, carried out

Received by the editors June 28, 2010 and, in revised form, February 24, 2011.

2010 Mathematics Subject Classification. Primary 35R35, 35J60, 35B65. 
in [5, is to show that whenever the free boundary is flat it is actually a Lipschitz graph in a small ball. Finally, in [3, it is shown that Lipschitz free boundaries are $C^{1, \alpha}$.

The major difference between this paper and Caffarelli's is work in the proof of the flatness. To the authors' knowledge, the only widely spread technique to prove flatness of the free boundary is, as in [4, to exploit non-degeneracy and regularity of the solution. In this case we have neither non-degeneracy nor optimal regularity. Instead, in section 4, we use an argument from geometric measure theory to deduce that the free boundary is flat almost everywhere with respect to the measure $\mu=\Delta u^{+}$, which of course lives on the free boundary. This is somewhat weaker than the result in [4, which states that the free boundary has finite $(n-1)$-dimensional Hausdorff measure and that the free boundary is flat almost everywhere with respect to that measure. We are only able to show that the free boundary is $\sigma$-finite with respect to the $(n-1)$-dimensional Hausdorff measure. Even though our result is somewhat weaker than what one usually gets in these kinds of problems, we believe that our flatness result is the most interesting new result in this article.

The second and third steps in the above strategy very closely follow the proofs in [5] and [3, and we provide little new mathematics in sections 5-7. However, for several reasons we have chosen to include the arguments in some detail. First, there have been a number of publications extending the results in [5] and [3] in many different directions; anisotropic problems in [9] and non-linear problems in [12, [13] and [14] for instance. In this literature there has been a standing refrain that the results can be extended to functions $G$ that depend on the normal of the free boundary, usually without proof. This is prudent in the situations in which those authors are interested, which is in general to extend the class of operators in which Caffarelli's method applies. In our case though, the dependence of the normal of the free boundary in the free boundary condition is essential, so we believe that it is important to explicitly work this case through.

Second, it is often assumed that $G(0)>0$, which amounts to a non-degeneracy in the free boundary condition. This assumption is important for the non-degeneracy that is usually needed to prove flatness properties of the free boundary. Here we do not have that assumption, so we need to verify that the proofs work even in this case. In one article our situation was even explicitly excluded.

Therefore we will provide the reader with some details in sections 6 and 7 of how flatness leads to Lipschitz regularity and how Lipschitz regularity leads to $C^{1, \alpha}$ regularity of the free boundary. We have judged it to be unnecessary to give proofs for all the lemmas presented in those sections, in particular lemmas where explicit proofs, as opposed to the standard refrain stating that the results can be extended to dependence of the normal of the free boundary in the free boundary condition, can be found in other publications. There is some redundancy though, since our proofs are quite similar to those that can be found in other places. We have also deemed it necessary to give enough details so the non-expert reader can see how the string of lemmas fit together to constitute a proof of our main result, which is as follows.

Theorem 1.1. Let $u$ be a solution to $\operatorname{div}(B \cdot \nabla u)=0$, where $B=B(u)=I+$ $(A-I) \chi_{\{u<0\}}, \chi_{\{u<0\}}$ denotes the characteristic function of the set $\{x: u(x)<0\}$ and $A$ is a matrix with strictly positive and bounded eigenvalues. 
Then $\mu=\Delta u^{+}$is a positive and locally finite measure whose $\operatorname{spt}(\mu) \subset F$ and has $\sigma$-finite $(n-1)$-dimensional Hausdorff measure. Furthermore for $\mu$-a.e. $x \in F$ there is a ball $B_{r}(x)$ such that $F \cap B(x)$ is a $C^{1, \alpha}$ graph.

In the next section we will describe how this result relates to the Bellman equations and formulate the main theorem in this context. In section 3 we will do some preliminary calculations. In section 4 we will prove that for $\mu$ almost every $x \in \Gamma$ there is a tangent measure of $\mu$ at $x$ whose support is contained in a hyperplane. Then we remind ourselves of some Harnack inequalities that we need in sections 6 and 7 , where we prove that the free boundary is Lipschitz and then $C^{1, \alpha}$ almost everywhere. Then in the final section we prove the main result.

\section{An application to the Bellman equations}

The authors' main interest in studying these equations is that they are strongly related to the Bellman equation

$$
F\left(D^{2} w\right)=\inf _{\alpha \in S}\left(L^{\alpha} w\right)=0,
$$

where $S$ is some index set and $L^{\alpha} w$ is an elliptic linear second order partial differential operator. Solutions are naturally defined in the viscosity sense, but L.C. Evans (see [8]) has proved the existence of classical $C^{2, \beta}$ solutions under mild conditions on $L^{\alpha}$. In particular, if $L^{\alpha}$ is a uniformly elliptic constant coefficient partial differential equation, then the solution is in $C^{2, \beta}$.

We will restrict ourselves to the simplest example of a Bellman equation,

$$
F\left(D^{2} w\right)=\inf (\Delta w, L w)=0,
$$

where $L$ is a constant coefficient uniformly elliptic equation

$$
L w=\Sigma_{i, j=1}^{n} a^{i j} \frac{\partial^{2} w}{\partial x_{i} \partial x_{j}} .
$$

Let us denote $u^{+}=L w \geq 0$ and $u^{-}=\Delta w \geq 0$ and $u=u^{+}-u^{-}$, and then, by Evans regularity result, $u \in C^{\beta}$. Moreover, $\Delta u=0$ in $\Omega^{+}=\{x ; u(x)>0\}$ and $L u=0$ in $\Omega^{-}=\{x ; u(x)<0\}$. In this section we will prove that $u^{ \pm}$will satisfy an energy balance condition across its zero set and that $u$ is a solution to the free boundary problem described in the previous section. By the definition of $u^{ \pm}$we will have $\Delta w=0$ in $\Omega^{+}$and $L w=0$ in $\Omega^{-}$. The free boundary, $F=\{x ; u(x)=0\}$, is the set where $w$ changes from being a solution to $\Delta w=0$ to becoming a solution to $L w=0$.

In the next two lemmas we show that the function $u$ is actually a solution to the free boundary problem described in section 1 .

Lemma 2.1. Let $\mu=\Delta u^{+}$and $\lambda=L u^{-}$. Then $\mu$ and $\lambda$ are locally finite measures and $\mu=\lambda$.

Proof. That $\mu$ and $\lambda$ are measures is easy to see using the fact that $u \geq 0$ and $\Delta u=0$ in $\{u>0\}$, and thus $u$ satisfies the sub-meanvalue property. Since $u$ is bounded it follows that $\mu$ is locally bounded. A similar argument holds for $\lambda$.

Let us consider the distribution $D: C^{4} \rightarrow \mathbf{R}$ :

$$
D(v)=\int_{B_{1}} w \Delta L v .
$$


Then

$$
D(v)=\int_{B_{1}} w \Delta L v=\int_{B_{1}} u^{-} L v=\int_{B_{1}} v d \lambda .
$$

This means that $D(\cdot)$ is a positive distribution and we can extend it to $C\left(B_{1}\right)$, and therefore it has a unique representation as a measure.

Also,

$$
D(v)=\int_{B_{1}} w \Delta L v=\int_{B_{1}} w L \Delta v=\int_{B_{1}} u^{+} \Delta v=\int_{B_{1}} v d \mu .
$$

It follows that $\mu=\lambda$.

In what follows it will be convenient to denote $B=I+(A-I) \chi_{\{u<0\}}$, where $A$ is the matrix $\left(a^{i j}\right)$ and $I$ the identity matrix.

Lemma 2.2. The function $u$ is a weak solution to $\operatorname{div}(B \cdot \nabla u)=0$.

Proof. Let $\phi \in C_{0}^{\infty}$. Then, by integration by parts and Lemma 2.1,

$$
\begin{aligned}
\int_{B_{1}} \nabla \phi \cdot B \cdot \nabla u & =\int_{B_{1}} \nabla \phi \cdot B \cdot \nabla u^{+}-\int_{B_{1}} \nabla \phi \cdot B \cdot \nabla u^{-} \\
& =\int_{B_{1}} \phi d \mu-\int_{B_{1}} \phi d \lambda=0 .
\end{aligned}
$$

In the context of Bellman equations our main theorem can be stated as follows.

Theorem 2.3. Let $w$ be a solution to $\inf (\Delta w, L w)=0$. Then $L \Delta w=\mu$, where $\mu$ is a locally bounded measure. Moreover,

(1) for $\mu$ almost every point $y \in \operatorname{spt}(\mu)$ there is a ball $B_{r}(y)$ such that the support of $\mu$ is a $C^{1, \alpha}$ graph in $B_{r}(y)$,

(2) the support of $\mu$ has $\sigma$-finite $(n-1)$-dimensional Hausdorff measure.

\section{Preliminary considerations}

In this section we will make some preliminary observations relating to the free boundary and the behavior of the solution close to free boundary points.

One standard tool in analyzing the free boundary is by blow-up, defined in the next definition.

Definition 3.1. Let $u$ be a function. Then $u_{r, x^{0}}$ will denote the rescaled and re-normalized function according to

$$
u_{r, x^{0}}(x)=\frac{u\left(r x+x^{0}\right)}{\|u\|_{L^{2}\left(B_{r}\left(x^{0}\right)\right)}} .
$$

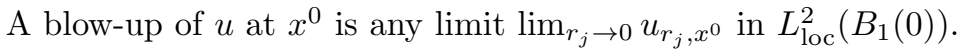

We will often not indicate the point $x^{0}$ if $x^{0}=0$ and just write $u_{r}$ for $u_{r, x^{0}}$.

In what follows we will pay extra attention to free boundary points where the free boundary is flat; that is, points where the blow-up is a two plane solution defined as follows.

Definition 3.2. We say that a solution $u$ is a two plane solution if $u$ is of the form $u=\alpha(\eta \cdot x)_{+}-\beta(\eta \cdot x)_{-}$, for some $\alpha, \beta>0$ and some vector $\eta \in \mathbf{R}^{n}$.

Our first lemma is a crude observation that states that for non-trivial solutions the free boundary has no interior. 
Lemma 3.3. Assume that there is an open set $\Sigma \subset B_{1}$ such that if $u^{+}=u^{-}=0$ in $\Sigma$, then $u^{ \pm}=0$ in $B_{1}$.

Proof. If such a set exists, then $L u^{-}=0$ in $\operatorname{int}\left(\overline{\Sigma \cup \Omega^{+}}\right)$; thus $\Delta u^{+}=0$ in $\operatorname{int}\left(\overline{\Sigma \cup \Omega^{+}}\right)$since $\lambda=\mu$. But then $u^{+}$is a non-negative harmonic function that is identically zero on an open set of its domain, and thus $u^{+}=0$ by the maximum principle; similarly for $u^{-}$.

The next simple lemma states that the measure $\mu$ controls the growth of $u$ away from the free boundary. The right inequality is true for every non-negative solution to $u \Delta u=0$, but in the left inequality we need the fact that our function is a solution to our free boundary problem.

Lemma 3.4. Let $x^{0} \in \Gamma \cap B_{1 / 2}$. Then for $r<1 / 2$ we have

$$
c r^{n-2} \sup _{B_{r}\left(x^{0}\right)} u^{+} \leq \mu\left(B_{2 r}\left(x^{0}\right)\right) \leq C r^{n-2} \sup _{B_{4 r}\left(x^{0}\right)} u^{+} .
$$

Proof. We may assume that $x^{0}=0$. Since $0 \in \Gamma$ we know that $u(0)=0$, and by Green's representation we can write (in this calculation $c$ will denote some dimensional constant that may differ on each occasion)

$$
\begin{gathered}
0=u^{+}(0)=-\int_{B_{4 r}}\left(\frac{c}{|x|^{n-2}}-\frac{c}{(4 r)^{n-2}}\right) d \mu+\frac{c}{r^{n-1}} \int_{\partial B_{4 r}} u^{+} \\
\leq-\frac{c}{r^{n-2}} \int_{B_{2 r}} d \mu+\frac{c}{r^{n-1}} \int_{\partial B_{4 r}} u^{+} \leq-c r^{2-n} \mu\left(B_{2 r}\right)+c \sup _{\partial B_{4 r}} u^{+} .
\end{gathered}
$$

Using the fact that $u^{+}$is subharmonic and thus $\sup _{B_{4 r}} u^{+} \leq \sup _{\partial B_{4 r}} u^{+}$, the right inequality follows.

To show the left inequality we argue by contradiction. Assume that there exists a sequence $u_{j}$ of solutions such that $\sup _{B_{r_{j}}} u_{j}^{+}=k_{j}$ and $\mu_{j}\left(B_{2 r_{j}}\right) \leq k_{j} / j$. By a rescaling and renormalization we may assume that $k_{j}=r_{j}=1$. By subharmonicity we will have $\left\|u_{j}^{+}\right\|_{L^{1}\left(B_{3 / 2}\right)} \geq C$, and by the formula

$$
0=u_{j}(0)=\frac{c}{s^{n-1}} \int_{\partial B_{s}} u_{j}^{+}-c \int_{B_{s}} \frac{1}{|x|^{n-2}}-\frac{1}{s^{n-2}} d \mu_{j}
$$

and by $\mu_{j}\left(B_{2}\right) \leq 1 / j$ we may deduce that $\left|\frac{c}{s^{n-1}} \int_{\partial B_{s}} u_{j}^{+}-\frac{c}{t^{n-1}} \int_{\partial B_{t}} u_{j}^{+}\right| \leq C_{j} \rightarrow 0$, for $1 / 2 \leq s, t \leq 7 / 4$. This implies that $\left\|u_{j}^{+}\right\|_{L^{1}\left(B_{3 / 2}\right)} \leq C\left\|u_{j}^{+}\right\|_{L^{1}\left(B_{1}\right)}$.

Therefore $u_{j}^{+} /\left\|u_{j}^{+}\right\|_{L^{1}\left(B_{3 / 2}\right)}$ will converge, in $L_{\text {loc }}^{1}$, to a positive non-zero harmonic function $u_{0}$. But $w_{j}=1-u_{j}$ is also a solution to $\operatorname{div}\left(B\left(u_{j}\right) \nabla w_{j}\right)=0$; moreover, $w_{j}(0)=1$ and $w_{j} \geq 0$ in $B_{1}$. By the Harnack inequality we get $w_{j} \leq C$ in $B_{3 / 4}$, and by standard regularity theory for weak solutions $w_{j} \in C^{\alpha}\left(B_{1 / 2}\right)$ uniformly; in particular, $v_{j} \in C^{\alpha}\left(B_{1 / 2}\right)$. So, in fact, $u_{j}$ will converge pointwise uniformly to $u_{0}$. But $u_{0}(0)=\epsilon>0$, since $u_{0}$ is a positive harmonic function. So for $j$ large enough $u_{j}(0)>\epsilon / 2>0$, which contradicts the fact that the origin is a free boundary point.

For the next lemma we need the H. W. Alt, L. C. Caffarelli and A. Friedman monotonicity formula; see [1], and for the last statement in the lemma see [6]. 
Lemma 3.5. Let $h_{1}$ and $h_{2}$ be continuous non-negative harmonic functions with disjoint support such that $h_{1}(0)=h_{2}(0)=0$. Then the following functional, $\Psi$, is monotonously non-decreasing in $r$ :

$$
\Psi\left(r, h_{1}, h_{2}\right)=\frac{1}{r^{4}} \int_{B_{r}} \frac{\left|\nabla h_{1}\right|^{2}}{|x|^{n-2}} \int_{B_{r}} \frac{\left|\nabla h_{1}\right|^{2}}{|x|^{n-2}} .
$$

Moreover, $\partial_{r} \Psi=0$ only if either $h_{1}=0, h_{2}=0$ or $h_{i}$ is harmonic in its support and $\operatorname{spt}\left(h_{i}\right) \cap B_{r}$ equals a spherical cap for $i=1,2$.

In the next lemma we use the monotonicity formula to show that whenever the free boundary is regular from one side it is actually flat at that point. This lemma will be useful in allowing us to use some ideas from geometric measure theory in our general flatness proof in section 4 .

Lemma 3.6. If $B_{\delta}(y) \subset \Omega^{-}$or $B_{\delta}(y) \subset \Omega^{+}$, and $x^{0} \in \overline{B_{\delta}(y)} \cap F$, then the blow-up of $u$ at $x^{0}$ is a unique two plane solution.

Proof. We will prove this lemma for $B_{\delta}(y) \subset \Omega^{-}$. In the case $B_{\delta}(y) \subset \Omega^{+}$, then we apply the same proof to $-u(\sqrt{A} x)$.

By translating, rotating and rescaling we may assume that $x^{0}=e_{n}$ and that $B_{\delta}(y)=B_{1}(0)$. Since $\Omega^{-}$is regular from the inside, $\sup _{B_{r}\left(e_{n}\right)} u^{-} \geq \epsilon r$ for some small $\epsilon>0$. We need to show a similar estimate for $u^{+}$. Then we can use the final statement in the monotonicity formula to deduce that $\lim _{r \rightarrow 0} u\left(r x+e_{n}\right) / r=u^{0}$, where $u^{0}$ is a unique two plane solution.

By a simple barrier argument, using the fact that $\omega^{+}$is regular from the outside, we see that $\sup _{B_{r}\left(e_{n}\right)} u^{+} \leq C r$ since $\Omega^{+}$. Going for a contradiction we assume that there exists a sequence of $r_{j} \rightarrow 0$ such that $\sup _{B_{r_{j}}\left(e_{n}\right)}\left(u^{+}\right) / \sup _{B_{r_{j}}\left(e_{n}\right)}\left(u^{-}\right) \rightarrow 0$. Then $u\left(r_{j} x+e_{n}\right) / \sup _{B_{r_{j}}} u^{-}$will converge (in $C^{\beta}$ ) to a non-positive function $u^{0}$, solving $\operatorname{div}\left(B \cdot \nabla u^{0}\right)=0$. By the Harnack inequality for $L$ we may deduce first that $u^{0} \neq 0$ and then that $u^{0}<0$ in $B_{1 / 2}$, contradicting the fact that $u(0)=0$.

This in particular implies that $\sup _{B_{r}\left(e_{n}\right)} u^{ \pm} / r$ is bounded from above and below uniformly for small $r$. Thus $u(r x) / r$ will have a convergent sub-sequence as $r \rightarrow 0$, and the limit $u^{0}$ will be linearly bounded: $c r \leq \sup _{B_{r}}\left(u^{0}\right)^{ \pm} \leq C r$. Moreover, $\operatorname{spt}\left(\left(u^{0}\right)^{+}\right) \subset \mathbb{R}_{+}^{n}$.

To conclude the lemma we define $v$ by the Kelvin transform of $u^{+}$; that is, $v(x)=u^{+}\left(x /|x|^{2}\right)$. Then $v$ is harmonic in $B_{1 / 2}\left(e_{n}\right)$ and $\operatorname{spt}(v) \subset B_{1}$, and also $v$ will inherit the linear growth of $u^{+}$away from $e_{n}$. In particular, we may apply the monotonicity formula to $u^{+}$and $v\left(u^{+}=h_{1}\right.$ and $v=h_{2}$ in Lemma 3.5.).

Observe that $\Psi\left(r, u^{+}\left(x+e_{n}\right), v\left(x+e_{n}\right)\right)=\Psi\left(1, u^{+}\left(r x+e_{n}\right) / r, v\left(r x+e_{n}\right) / r\right)$. This implies that

$$
\lim _{r \rightarrow 0} \Psi\left(r, u^{+}\left(x+e_{n}\right), v\left(x+e_{n}\right)\right)=\lim _{r \rightarrow 0} \Psi\left(1, u^{+}\left(r x+e_{n}\right) / r, v\left(r x+e_{n}\right) / r\right) .
$$

By the non-degeneracy of $u^{+}$and $v$ and the monotonicity of $\Psi$, we may conclude that the $\operatorname{limit}_{\lim _{r \rightarrow 0}} \psi\left(1, u^{+}\left(r x+e_{n}\right) / r, v\left(r x+e_{n}\right) / r\right)$ converges to a fixed non-zero value. By the last statement in Lemma 3.5 it follows that $\operatorname{spt}\left(u^{+}\right)$is a half-space. Therefore, being a positive harmonic function in a half-space $u^{+}=\alpha x_{n}$ for some $\alpha>0$.

Therefore $u\left(r x+e_{n}\right) / r \rightarrow \alpha\left(x_{n}\right)^{+}+\beta\left(x_{n}\right)^{-}$. Since $\sup _{B_{r}}|u| \approx r$ and $u(r x) / r$ converge to a two plane solution, it follows that the blow-up $u\left(r x+e_{n}\right) /\|u\|_{L^{2}\left(B_{r}\left(e_{n}\right)\right)}$ converges to a two plane solution. 


\section{THE FREE BOUNDARY IS FLAT}

In this section we will show that the free boundary is flat at $\mu$ almost everywhere. To be more precise we will show that for $\mu$ a.e. $x^{0} \in F$ there exists a sequence $r_{j} \rightarrow 0$ such that $u_{r_{j}, x^{0}} \rightarrow P$, where $P$ is a two plane solution.

This will naturally imply that around $\mu$ almost every point of the free boundary, the free boundary is flat at some scale. That is enough to use the machinery of Caffarelli to show that the free boundary is actually Lipschitz and $C^{1, \alpha}$ around those points.

The argument uses some ideas to prove rectifiability of measures, [1] or [7. Corresponding to the set of tangent measures in geometric measure theory we have the following definition.

Definition 4.1. $B l o(u, a)$ or, if $u$ is given by the context, just $B l o(a)$ will stand for the set of all possible blow-up limits of $u$ at the point $a \in F$.

The next proposition shows that blow-ups of blow-ups at a point $a$ are also in $B l o(a)$, at least $\mu$ almost everywhere.

Proposition 4.2. For $\mu$-a.e. a the following holds. Let $u^{a} \in B l o(a)$ and $x^{a} \in$ $F_{u^{a}}$; then $u_{x^{a}, r}^{a} \in B l o(u, a)$ for every $r>0$.

Proof. Let

$$
\begin{aligned}
A_{k, j}= & \left\{a \in \mathbf{R}^{n} ; \exists u_{0} \in \operatorname{Blo}(a) \text { and an } x^{a} \in \Gamma_{u_{0}}\right. \text { such that } \\
& \left.\left\|u_{r, a}-u_{1, x^{a}}^{a}\right\|_{L^{2}\left(B_{1}(0)\right.} \geq \frac{1}{k}, \text { for all } r<\frac{1}{j}\right\} .
\end{aligned}
$$

We will show that $\mu\left(A_{k, j}\right)=0$.

Denote $S=\left\{u_{1, x^{a}}^{a}\right\}_{a \in A_{k, j}}$, where $u_{1, x^{a}}^{a}$ are considered to be functions in $W^{1,2}\left(B_{1 / 2}\right)$. $S$ is a bounded set in $W^{1,2}\left(B_{1 / 2}\right)$ and therefore compact in $L^{2}\left(B_{1 / 2}\right)$. Cover $S$ by $G_{i}$, where $G_{i}=\left\{w ;\left\|w-w_{i}\right\|_{L^{2}}<\frac{1}{4 k}\right\}$ for some countable family of functions $w_{i}$.

If $\mu\left(A_{k, j}\right)>0$, then we will have $\mu\left(\left\{a \in A_{k, j} ; u_{1, x^{a}}^{a} \in G_{i}\right\}\right)>0$ for at least one

$i$. We will denote $A=\left\{a \in A_{k, j} ; u_{1, x^{a}}^{a} \in G_{i}\right\}$ for that, or one of those, $i$.

Then

$$
\mu(A)>0 .
$$

(2) If $a \in A$, then there exists $u^{a} \in B l o(a)$ with

$$
\left\|u_{a, r}-u_{1, x^{a}}^{a}\right\|_{L^{2}}\left(B_{1 / 2}\right) \geq \frac{1}{k} \text { for all } r<\frac{1}{j}
$$

(3) and

$$
\left\|u_{1, x^{a}}^{a}-u_{1, x^{b}}^{b}\right\|_{L^{2}}<\frac{1}{2 k}
$$

for every $a, b \in A$.

We will also choose

(4) $a \in A$ such that

$$
\lim _{r \rightarrow 0} \frac{\mu\left(A \cap B_{r}(a)\right)}{\mu\left(B_{r}(a)\right)}=1,
$$

(5) $r_{i} \rightarrow 0$ such that $u_{r_{i}, a} \rightarrow u^{a}$ weakly in $W^{1,2}\left(B_{1 / 2}\right)$, 
(6) $a_{i} \in A$ such that

$$
\left|a_{i}-a+r_{i} x^{a}\right|<\operatorname{dist}\left(a+r_{i} x^{a}, A\right)+\frac{r_{i}}{i} .
$$

That we can make the choice satisfying point (4). follows from the fact that a.e. point is a point of density and (5) follows from weak compactness.

For (6) we see that if $\lim _{i \rightarrow \infty} \frac{\operatorname{dist}\left(a+r_{i} x^{a}, A\right)}{r_{i}}=\epsilon>0$, then $\mu_{u^{a}}\left(B_{\epsilon}\left(\left(x^{a}\right)\right)\right)=0$ and also $u^{a}\left(x^{a}\right)=0$. Therefore $\left(u^{a}\right)^{+^{2}}=0$; also by Lemma $3.3\left(u^{a}\right)^{-}=0$, so $u^{a}=0$, and therefore the proposition is trivially true. So we may assume that $\lim _{i \rightarrow \infty} \frac{\operatorname{dist}\left(a+r_{i} x^{a}, A\right)}{r_{i}}=0$, and we can thus satisfy (6) by choosing a sub-sequence in $r_{i}$.

Next we note that

$$
u_{r_{i}, a_{i}}=\left(u_{r_{i}, a}\right)_{1,\left(a_{i}-a\right) / r_{i}} \rightarrow u_{1, x^{a}}^{a}
$$

weakly in $W^{1,2}$ and thus strongly in $L^{2}$. Therefore

$$
\left\|u_{1, x^{a}}^{a_{i}}-u_{r_{i}, a_{i}}\right\|_{L^{2}}<\frac{1}{2 k}
$$

since $A=\left\{a \in A_{k, j} ; u_{1, x^{a}}^{a} \in G_{i}\right\}$. Also

$$
\left\|u_{r_{i}, a_{i}}-u_{1, x^{a_{i}}}^{a_{i}}\right\|_{L^{2}} \geq \frac{1}{k}
$$

if $r_{i}<1 / j$ since $A \subset A_{k, j}$.

By the triangle inequality we have

$$
\left\|u_{1, a_{i}}^{a_{i}}-u_{r_{i}, a_{i}}\right\|_{L^{2}} \leq\left\|u_{r_{i}, a_{i}}^{a_{i}}-u_{1, x^{a}}^{a}\right\|_{L^{2}}+\left\|u_{1, x^{a}}^{a}-u_{r_{i}, a_{i}}\right\|_{L^{2}} .
$$

We estimate the first term to the right by equation (4.3) and the second term by equation (4.6) and deduce

$$
\left\|u_{1, a_{i}}^{a_{i}}-u_{r_{i}, a_{i}}\right\|_{L^{2}}<\frac{1}{k}
$$

but this contradicts equation (4.7), and the lemma follows.

To be able to use the previous proposition we need to have some control over the set where $\mu$ is very small or, equivalently, where $u$ is very degenerate. The following crude lemma establishes what we need.

Lemma 4.3. Let $D_{k}(\mu)=\left\{x^{0} \in \operatorname{spt}(\mu) ; \lim \sup _{r \rightarrow 0} \frac{\mu\left(B_{r}\left(x^{0}\right)\right)}{r^{k}}<\infty\right\}$; then for every $k>n$ the measure $\nu_{k}=\left.\mu\right|_{D_{k}}$ satisfies $\nu_{k}\left(B_{1}\right)=0$. Here $\left.\mu\right|_{A}$ is the restriction of $\mu$ to the set $A$.

Proof. Obviously $\lim \sup _{r \rightarrow 0} \frac{\nu_{k}\left(B_{r}\left(x^{0}\right)\right)}{r^{k}}<\infty$, which means that $\nu_{k}$ is absolutely continuous with respect to $\mathbb{H}^{k}$, the $k$-dimensional Hausdorff measure. Since $\mathbb{H}^{k}\left(B_{1}\right)=0$ the lemma follows.

Now we are ready to prove the main result of this section. The idea is that for $\left.\mu\right|_{F \backslash D_{n+1}}$ almost every point, $y \in F$, we can find a blow-up limit that is not identically zero. But since the limit is not zero there must be a free boundary point that is touchable by a ball, and thus by Lemma 3.6 there is a blow-up of the blow-up that is a two plane solution and therefore there is (for a.e. point $y \in F$ ) a blow-up sequence converging to a two plane solution. 
Proposition 4.4. For $\mu$-a.e. point $x^{0} \in F$ there exists a sequence $r_{j} \rightarrow 0$ such that

$$
u_{r_{j}, x^{0}} \rightarrow u^{0}
$$

where $u^{0}$ is a two plane solution.

Proof. We will show that for $\mu$ almost every $x^{0} \in F \backslash D_{n+1}$ there exists a blow-up sequence $u_{r_{j}, x^{0}}$ converging to a two plane solution. Since $\left.\mu\right|_{D_{n+1}}=0$ by Lemma 4.3. this would prove the proposition.

Fix an $x^{0} \in F \backslash D_{n+1}$ and choose a sequence $r_{j} \rightarrow 0$ such that $\mu\left(B_{r}\left(x^{0}\right)\right) / r^{n+1} \leq$ $\mu\left(B_{r_{j}}\left(x^{0}\right)\right) / r_{j}^{n+1}$ for all $r>r_{j}$. Such a choice is possible since

$$
\limsup _{r \rightarrow 0} \mu\left(B_{r}\left(x^{0}\right)\right) / r^{n+1}=\infty .
$$

Make the blow-up

$$
v^{j}(x)=u^{j}\left(r_{j} x+x^{0}\right) r^{n-2} / \mu\left(B_{r_{j}}\left(x^{0}\right)\right) .
$$

From Lemma 3.4 and $\mu_{j}\left(B_{r}\right) \leq C\left(1+r^{n+1}\right)$ we know that $v^{j}$ is locally bounded and non-degenerate; here $\mu_{j}$ is the measure $\Delta\left(v^{j}\right)^{+}$. Therefore, for a sub-sequence, $v^{j} \rightarrow v^{0}$ locally in $C^{\beta}$. Technically $v^{0}$ is not a blow-up of $u$ since we divide by $\mu\left(B_{r_{j}}\left(x^{0}\right)\right)$ and not the $L^{2}$-norm in equation (4.8), but since $v^{0}$ is non-degenerate it only differs by a constant from a "real" blow-up so we do not care about the slight abuse of vocabulary.

It is easy to see that there exists a ball $B_{r}(y) \subset\left\{v^{0}>0\right\}$ touching the levelset $\left\{v^{0}=0\right\}$ at some point $z$. By Lemma 3.6 the blow-up of $v^{0}$ at $z$ is a two plane solution, and therefore by Proposition 4.2 there exists a two plane solution in $B l o\left(x^{0}\right)$.

\section{HARNACK INEQUALITIES}

The next two sections will rely heavily on Harnack inequalities in different forms. In this section we have gathered those without proofs. The first one is classical and a proof can be found in any good book on elliptic PDE, for instance [10.

Lemma 5.1. Let $u$ be a non-negative harmonic function $i B_{1}$. Then

$$
\sup _{B_{r}} u \leq C(r) \inf _{B_{r}} u
$$

Proofs of the following two Harnack inequalities can be found in 2, which is also an excellent reference on the theory in the next two sections.

Lemma 5.2. Let $u$ and $v$ be non-negative harmonic functions in $B_{1} \cap\left\{x_{n} \geq f\left(x^{\prime}\right)\right\}$ where $f$ is a Lipschitz function satisfying $f(0)=0$ and $|\nabla f| \leq \delta \leq 1 / 4$. Assume furthermore that $u\left(x^{\prime}, f\left(x^{\prime}\right)\right)=v\left(x^{\prime}, f\left(x^{\prime}\right)\right)=0$ and $u\left(e_{n} / 2\right)=v\left(e_{n} / 2\right)=1$. Then $u / v \in C^{\alpha}\left(B_{1 / 2} \cap\left\{x_{n} \geq f\left(x^{\prime}\right)\right\}\right)$ and $\|u / v\|_{C^{\alpha}\left(B_{1 / 2} \cap\left\{x_{n} \geq f\left(x^{\prime}\right)\right\}\right)} \leq C(n, \delta)$.

In the next lemma we use the notation $\Gamma\left(\theta, e_{n}\right)=\left\{\tau \in \mathbb{R}^{n} ; \tau \cdot e_{n} \geq \theta\right\}$ (see Definition 6.2).

Lemma 5.3. There exist positive constants $\bar{c}$ and $\mu$, depending only on $\theta_{0}$ and $n$, such that for each small vector $\tau \in \Gamma \frac{\theta}{2}, e_{n}$ and $x \in B_{1 / 8}$ we have

$$
\sup _{y \in B_{(1+\mu b) \epsilon}(x)} u(y-\tau) \leq u(x)-\bar{c} b \epsilon u\left(x_{0}\right) .
$$




\section{Flat FREE BOUNDARIES ARE LiPSCHITZ}

In this section we will almost word for word remind ourselves of the regularity theory of Caffarelli [5]. Another good resource for the material in this section is the book 2. We will also use some results from Feldman [9], who extended Caffarelli's ideas to non-isotropic problems.

The general idea is that if $u$ is close to a two plane solution $P\left(x_{n}\right)$ in $Q_{1}$, then a small translation $v(x)=u\left(x \pm \epsilon x_{n}\right)$ should become smaller than $u$. We also expect some regularization; that is, that in $Q_{1 / 2}$ we expect that even a smaller translation is enough to make the translated function smaller. In reality it is hard to work with translations, so we will be working with the related concept of sup-convolutions.

In particular, we would expect that

$$
\sup _{y \in B_{\delta}(x)} v(y) \leq u(x) \quad \text { in } Q_{1 / 2} \text { for some } \delta>0
$$

To prove this we use a comparison for our solutions (Lemma 6.4). To use the comparison principle we naturally need to take the boundary values into consideration, so instead of proving equation (6.9) we will try to prove $\sup _{y \in B_{\phi(x)}(x)} v(y) \leq u(x)$ for some good choice of $\phi$. In particular, we would want $\phi$ to be large in $Q_{1 / 2}$. To be able to use comparison we also need the expression to the left to be a subsolution. In Lemma 6.5 we find a condition that makes $\sup _{y \in B_{\phi(x)}(x)} v(y)$ into a sub-solution away from the free boundary, and in Lemma 6.6 we choose $\phi$. We also need $\sup _{y \in B_{\phi(x)}(x)} v(y)$ to be a sub-solution at the free boundary. In Lemma 6.7 we investigate what free boundary condition $\sup _{y \in B_{\phi(x)}(x)} v(y)$ satisfies, and then in Lemma 6.9 we make a small perturbation of $\sup _{y \in B_{\phi(x)}(x)} v(y)$ to assure that we get a sub-solution across the free boundary. Then in the rest of this section we show that equation (6.9) is in fact true, with $\delta$ depending on $x$, and that Lipschitz regularity of the free boundary follows.

As pointed out earlier, this section follows [5] (or the book [2]) very closely, and to more smoothly be able to use those results we will start by defining viscosity solutions to our free boundary problem. As a matter of fact our weak solutions are stronger than viscosity solutions, so all the theoretical framework of Caffarelli and Feldman applies to our problem.

Definition 6.1. We say that a continuous function $u$ is a viscosity sub-solution to our free boundary problem $\operatorname{div}(B(u) \cdot \nabla u)=0$ if

(1) $\Delta u \geq 0$ in $\Omega^{+}(u)=\{u>0\}$ and $L u \geq 0$ in $\Omega^{-}(u)=\{u<0\}$,

(2) if $B_{\epsilon}(y) \subset \Omega^{+}$and $x^{0} \in \overline{B_{\epsilon}(y)} \cap \overline{\Omega^{+}}$and $\nu$ is the normal of $\partial B_{\epsilon}(y)$ at $x^{0}$, then

$$
u(x) \geq \alpha\left(\nu \cdot\left(x-x^{0}\right)^{+}\right)-\beta\left(\nu \cdot\left(x-x^{0}\right)^{-}\right)+o(|x|),
$$

for some $\alpha \geq 0$ and $\alpha \geq \sqrt{\sum_{i, j=1}^{n} \nu_{i} \nu_{j} a^{i j}} \beta$, where $a^{i j}$ are the coefficients of $L$ as in section 1 .

A super-solution is defined similarly, with all the inequalities above reversed and the ball $B_{\epsilon}(y) \subset \Omega^{-}$.

A solution is any continuous function that is both a sub- and a super-solution. In particular, by Lemma 3.6. any weak solution to $\operatorname{div}(B(u) \cdot \nabla u)=0$ is a viscosity solution. 
As described in the introduction to this section it is natural to work with functions that decrease when translated slightly in a certain direction. The concept we will use is defined as follows.

Definition 6.2. We say that $u$ is $\epsilon_{0}$-monotone in the direction $\tau$ if $u(x+\epsilon \tau) \geq u(x)$ for every $\epsilon \geq \epsilon_{0}$.

We say that $u$ is $\epsilon_{0}$-monotone in the cone $\Gamma\left(\theta, e_{n}\right)=\left\{\tau ; \tau \cdot e_{n} \geq \theta\right\}$ if for any $\epsilon \geq \epsilon_{0}$ we have

$$
u(x) \geq \sup _{|y|<\sin (\theta)} u\left(x-\epsilon\left(e_{n}+y\right)\right)
$$

By the previous section our solution will be $\epsilon$-monotone around $\mu$ almost every free boundary point, as is formalized by the next lemma.

Lemma 6.3. Let $P(x)=P\left(x_{n}\right)$ be a two plane solution, $\|u\|_{L^{\infty}}\left(B_{1}\right)=1$ and $|u-P| \leq \delta / 2$. Then $u$ is $\epsilon-$ monotone in $\Gamma\left(\theta, e_{n}\right)$ for $\theta=\arcsin (1-\delta / \epsilon)$.

In particular, for every $\epsilon>0$ and $\theta<\pi / 2$ and for $\mu$ almost every $x^{0} \in F$ there is a ball $B_{r}\left(x^{0}\right)$ such that $u\left(r x+x^{0}\right) /\|u\|_{L^{\infty}}\left(B_{r}\left(x^{0}\right)\right)$ is $\epsilon$-monotone in $\Gamma(\theta, e)$ for some unit vector e.

Proof. The first part follows by simple verification. The second part is an easy consequence of the first part and Proposition 4.4.

The following lemma is taken directly from Feldman [9, Lemma 6].

Lemma 6.4. Let $v \leq u$ be two continuous functions in $\Omega, v<u$ in $\Omega^{+}(v)$, $v$ be a sub-solution and $u$ a solution. Let $x^{0} \in F(u) \cap F(v)$. Then $x^{0}$ cannot be touched by a ball in $\Omega^{+}(v)$; that is, there is no ball as in condition (2) in Definition 6.1 .

Idea of Proof. If such a ball exists, then $u-v$ would be a positive super-harmonic function in $\Omega^{+}(v)$, and therefore by Hopf's Lemma the normal derivative of $u-v$ at $x^{0}$ would be positive. This would contradict $v \leq u$ and the condition in (2) of Definition 6.1. For more details we refer the reader to 9 .

The next two lemmas will help us to construct what is almost a sub-solution to our free boundary problem. The first lemma is taken from Feldman [9], and for the one after we rely on [2].

Lemma 6.5. Let $N u=B^{i j} u_{i j}$ and $\phi>0$ be a $C^{2}$ solution to

$$
N \phi \geq \frac{C|\nabla \phi|^{2}}{\phi},
$$

where $C$ is a large constant. Let $u$ be a continuous function, defined in $B_{1+\|\phi\|_{L} \infty}$, satisfying $L u=0$ in $\{u>0\}$. Then $v(x)=\sup _{B_{\phi_{(x)}}(x)} u$ satisfies $L v \geq 0$ in $B_{1} \cap\{v>0\}$.

Lemma 6.6. For every $\gamma>0$ there is a family of functions $\phi_{t}$ in $Q_{1}, 0 \leq t \leq 1$, such that

(1) $1 \leq \phi_{t} \leq 1+t \mu$,

(2) $\phi_{t} N \phi_{t} \geq C\left|\nabla \phi_{t}\right|^{2}$,

(3) $\phi_{t}=1$ on $\left\{x, d\left(x, \partial Q_{1} \cap\left\{x_{n}=0\right\}\right)<\gamma\right\}$,

(4) $\phi_{t} \geq 1+t \frac{C \gamma\left(d\left(x, \partial Q_{1}\right)+\gamma\right)}{\left(d\left(x, \partial Q_{1} \cap\left\{x_{n}=0\right\}\right)+\gamma\right)^{2}}$ in $B_{1 / 8}$ and

(5) $\left|\nabla \phi_{t}\right| \leq C t / \delta$. 
Proof. There is only a slight difference between the proof of this lemma and Lemma 5.2 in [2. When the authors of [2] work with the Laplacian we need to work with Picci's extremal operators as in Lemma 3 in 13 .

Lemma 6.5 only states that the function $v$ is a sub-solution away from the free boundary, but to be able to use the comparison principle (Lemma 6.4) we need a full sub-solution that is also satisfying condition (2) in Definition 6.1. To get that we make a small perturbation of the solution in Lemma 6.9. but first we need to investigate how $v$ from Lemma 6.5 behaves at the free boundary. The following lemma (see Lemma 4.9 in [2]) takes care of that.

Lemma 6.7. Let $u$ and $v$ be as in Lemma 6.6. Assume furthermore that $x^{1} \in$ $\partial \Omega^{+}(v), y^{1} \in \partial \Omega^{+}(u)$ and $y^{1} \in \partial B_{\phi\left(x^{1}\right)}\left(x^{1}\right)$. Then

(1) $x^{1}$ is a regular point of the free boundary of $v$; that is, there exists a ball $B \subset \Omega^{+}(v)$ and $x^{1} \in \partial B \cap \partial \Omega^{+}(v)$.

(2) If $\nu=\left(y^{1}-x^{1}\right) /\left|y^{1}-x^{1}\right|$ and near $y^{1}$

$$
u(y)=\alpha\left(\left(y-y^{1}\right) \cdot \nu\right)^{+}-\beta\left(\left(y-y^{1}\right) \cdot \nu\right)^{-}+o\left(\left|y-y^{1}\right|\right),
$$

then

$v(x) \geq \alpha\left(x-x^{1}\right) \cdot\left(\nu+\nabla \phi\left(x^{1}\right)\right)^{+}-\beta\left(y-y^{1}\right) \cdot\left(\nu+\nabla \phi\left(x^{1}\right)\right)^{-}+o\left(\left|y-y^{1}\right|\right)$.

We also need a geometric result on the level sets of functions, directly quoted from [2].

Lemma 6.8. Let $u$ be $\epsilon-$ monotone in $\Gamma\left(\theta, e_{n}\right)$ and

$$
v(x)=\sup _{B_{\phi(x)}(x)} u \text {. }
$$

Then $v$ is monotone in the cone $\Gamma\left(\bar{\theta}, e_{n}\right)$ for every $\bar{\theta}$ satisfying

$$
\sin \bar{\theta} \leq \frac{1}{1+|\nabla \phi|}\left(\sin (\theta)-\frac{\epsilon}{2 \phi} \cos ^{2}(\theta)-|\nabla \phi|\right) .
$$

In particular, if such a $\bar{\theta}$ exists, then the level sets of $v$ are Lipschitz.

The next lemma establishes a comparison function for our free boundary problem. The proof of the lemma is very similar to what has been written before, but since we did not find any good reference, with normal dependence in the free boundary condition, we give a proof.

Lemma 6.9. Let $u,\|u\|_{L^{\infty}\left(Q_{1}\right)}$, be a solution to our free boundary problem in $Q_{1}$ and assume that $|u-P| \leq \delta / 2$ for some two plane solution $P(x)=P\left(x_{n}\right)$ and $1 \gg \epsilon \gg \delta$. We also let

(1) $\phi_{t}$ be the function from Lemma 6.6,

(2) $v_{t}=\sup _{B_{\sigma \phi_{t}(x)}(x)} u$,

(3) $w$ be a harmonic function in $\Omega^{+}\left(v_{t}\right) \cap\{x<C M \epsilon\}$, for some large constants $C$ and $M$, with boundary values

$$
w= \begin{cases}u & \text { on }\left\{x_{n}=C M \epsilon\right\} \cap \Omega^{+}\left(v_{t}\right), \\ 0 & \text { elsewhere on } \partial\left(\Omega^{+}\left(v_{t}\right) \cap\{x<C M \epsilon\}\right),\end{cases}
$$

(4) $\bar{v}_{t}=v_{t}+\eta w$.

If $\theta \geq \pi / 4, \eta \geq C \sigma / \delta$ with $\sigma / \delta<<1$, then $\bar{v}_{t}$ is a sub-solution of our free boundary problem in $Q_{1-C \epsilon} \cap\left\{x_{n}<C M \epsilon\right\}$. 
Proof. From Lemma 6.5 we know that $v_{t}$ is a sub-solution away from its zero level set. Also, by definition, $\Delta w=0$ in $\Omega^{+} \cap\left\{x_{n}<C M \epsilon\right\}$. Therefore we only need to verify that $\bar{v}_{t}$ satisfies condition (2) in Definition 6.1.

From Lemma 6.7, with $v_{t}=v$ we get that every point of the free boundary is regular and that

$$
v_{t}(x) \geq \alpha\left(x-x^{1}\right) \cdot\left(\nu+\sigma \nabla \phi\left(x^{1}\right)\right)^{+}-\beta\left(y-y^{1}\right) \cdot\left(\nu+\sigma \nabla \phi\left(x^{1}\right)\right)^{-}+o\left(\left|y-y^{1}\right|\right),
$$

close to $x^{1} \in \partial \Omega^{+}\left(v_{t}\right)$.

Using $\left|\nabla \phi_{t}\right| \leq C t / \delta$ we may conclude that

$$
v_{t} \geq \bar{\alpha}\left(\left(x-x^{1}\right) \cdot \bar{\nu}\right)^{+}-\bar{\beta}\left(\left(y-y^{1}\right) \cdot \bar{\nu}\right)^{-}+o\left(\left|y-y^{1}\right|\right),
$$

with the notation $\bar{\nu}=\left(\nu+\sigma \nabla \phi_{t}\left(x^{1}\right)\right) /\left|\nu+\sigma \nabla \phi_{t}\left(x^{1}\right)\right|, \bar{\alpha}=\left|\nu+\sigma \nabla \phi_{t}\left(x^{1}\right)\right| \alpha$ and $\bar{\beta}=\left|\nu-\sigma \nabla \phi_{t}\left(x^{1}\right)\right| \beta$.

Also, since $u$ is a solution, we have

$$
\alpha=\sqrt{\sum_{i j} a_{i j} \nu_{i} \nu_{j}} \beta .
$$

The function $\nu \rightarrow \sqrt{\sum_{i j} a_{i j} \nu_{i} \nu_{j}}$ is Lipschitz and $\left|\nabla \phi_{t}\right| \leq C t / \delta$. We may conclude that

$$
\bar{\alpha} \geq\left(\sqrt{\sum_{i j} a_{i j} \bar{\nu}_{i} \bar{\nu}_{j}}-\frac{C \sigma t}{\delta}\right) \bar{\beta} .
$$

This gives us that $v_{t}$ is almost a sub-solution across the free boundary. Our next goal is to show that the small perturbation $\bar{v}_{t}$ is actually a sub-solution. To do that we need a good comparison between $w$ and $v_{t}$, which we obtain by the boundary Harnack inequality, Lemma 5.2. For that we need to show that the boundary $\partial \Omega^{+}\left(v_{t}\right)$ is Lipschitz. According to Lemma 6.8, we only need to show that

$$
\frac{1}{1+\sigma\left|\nabla \phi_{t}\right|}\left(\sin (\theta)-\frac{\epsilon}{2 \sigma \phi_{t}} \cos ^{2}(\theta)-\sigma\left|\nabla \phi_{t}\right|\right)>0
$$

which follows from $\theta \geq \pi / 4$ and $\sigma / \delta<<1$.

Therefore, by the boundary Harnack inequality (Lemma 5.2) and $w \approx v_{t}$ on $\left\{x_{n}=C M \epsilon\right\}$ we get

$$
\frac{v_{t}}{w} \leq C \quad \operatorname{in} \Omega^{+}\left(v_{t}\right) \cap\left\{x_{n}<C M \epsilon / 2\right\} .
$$

In particular, this implies that $C \partial_{\bar{\nu}} w \geq \bar{\alpha}$.

So $\bar{v}_{t}$ satisfies the following inequality at its free boundary:

$$
\bar{v}_{t} \geq \tilde{\alpha}\left(\left(x-x^{1}\right) \cdot \bar{\nu}\right)^{+}-\bar{\beta}\left(\left(y-y^{1}\right) \cdot \bar{\nu}\right)^{-}+o\left(\left|y-y^{1}\right|\right),
$$

with $\tilde{\alpha}=(1+\eta / C) \bar{\alpha}$. Inserting this in equation (6.10) and using $\eta \geq C \sigma / \delta$ for a constant $C$ large enough, we may deduce that

$$
\tilde{\alpha} \geq\left(\sqrt{\sum_{i j} a_{i j} \bar{\nu}_{i} \bar{\nu}_{j}}\right) \bar{\beta},
$$

which is exactly the sub-solution free boundary condition.

The next lemma, and also the proposition that follows, can also be found in [2].

Lemma 6.10. Let $\epsilon$ be small and $u$ be an $\epsilon-$ monotone harmonic function in the $e_{n}$-direction in $B_{1}$. Then $D_{n} u(0) \geq C\left(u\left(\epsilon e_{n}\right)-u(0)\right)$. 
Proposition 6.11. Let $u$ be a solution to our free boundary problem in $Q_{1}$ and assume that $u$ is $\epsilon-$ monotone in $\Gamma\left(\theta, e_{n}\right)$ for some small $\epsilon$ and $\pi / 4<\theta_{0} \leq \theta \leq \pi / 2$ (in particular, it is enough that $u$ is close enough to a two plane solution $P\left(x_{n}\right)$ with $\left.\left\|P\left(x_{n}\right)\right\|_{L^{\infty}\left(Q_{1}\right)}=1\right)$. Then, if $0 \in F$, there exists $\lambda<1$, such that $u$ is $\lambda \epsilon-$ monotone in the cone of directions $\Gamma\left(\theta-C \epsilon^{1 / 4}, e_{n+1}\right)$ in the domain $B_{1-\epsilon^{1 / 8}}^{\prime}$.

Proof. Consider the function $u_{1}(x)=u\left(x-\lambda \epsilon e_{n}\right)$ for $0<1-\lambda<\sin (\pi / 4)$. Then by $\epsilon$-monotonicity

$$
\sup _{B_{\epsilon(\sin (\theta)-(1-\lambda))}(x)} u_{1}(x) \leq u(x)=u_{2}(x),
$$

since

$$
B_{\epsilon(\sin (\theta)-(1-\lambda))}\left(x-\lambda \epsilon e_{n}\right) \subset B_{\epsilon \sin (\theta)}\left(x-\epsilon e_{n}\right) .
$$

From Lemma $6.10 u$ is fully monotone (that is, $0-$ monotone) in the cone $\Gamma\left(\theta, e_{n}\right)$ in $\left\{x_{n}>\epsilon M\right\}$ for some constant $M$. Therefore,

$$
\sup _{\lambda \in \sin (\theta)} u_{1}(x) \leq u_{2}(x)
$$

for any $x$ such that $e_{n} \cdot x>\epsilon M$.

We want to find an intermediate radius $\sigma \phi_{t}$ such that

$$
\sup _{\sigma \phi_{t}}(x) u_{1} \leq u_{2}(x)
$$

in $B_{1-\epsilon^{1 / 4}} \cap\left\{\left|x_{n}\right| \leq \epsilon M\right\}$. Here $\phi_{t}$ is the function from Lemma 6.6 .

To be able to use the previous lemmas we will fix

$$
\begin{gathered}
\sigma=\epsilon(\sin (\theta)-(1-\lambda)), \\
\lambda \geq \frac{3-\sqrt{2}}{2}, \\
\eta=C \epsilon^{1 / 4}, \quad \gamma=\sqrt{\epsilon}, \text { and finally } \\
1+t \leq \frac{\lambda \sin (\theta)-C \epsilon^{1 / 4}}{\sin (\theta)-(1-\lambda)} .
\end{gathered}
$$

Then $\bar{v}_{t}$ (as in Lemma 6.9) satisfies $\bar{v}_{t} \leq u_{2}$ on $Q_{1-C \epsilon} \cap\left\{\left|x_{n}\right| \leq C M \epsilon\right\}$. To see this we notice that on $\left\{x_{n}=C M \epsilon\right\}$ for any $l_{1}<l_{2}<\lambda$,

$$
\sup _{B_{l_{1}}(x)} u_{1} \leq \sup _{B_{l_{2}}(x)} u_{1}-\frac{l_{2}-l_{1}}{C}\left|\nabla u_{1}(x)\right| \leq\left(1-\operatorname{sgn}\left(u_{2}\right) \frac{l_{2}-l_{1}}{C \epsilon}\right) u_{2}(x) .
$$

Here $\operatorname{sgn}\left(u_{2}\right)$ is the sign of $u_{2}$. We have also used the fact that

$$
\left|\nabla u_{1}(x)\right| \approx \frac{u_{1}(x)}{\operatorname{dist}\left(x, \partial \Omega^{+}\left(u_{1}\right)\right)}
$$

since $u_{1}$ is harmonic and $\Omega^{+}\left(u_{1}\right)$ is Lipschitz.

By choosing $l_{1}=\lambda \epsilon \sin (\theta)-C \epsilon^{1+1 / 4}$ and $l_{2}=\lambda \epsilon \sin (\theta)-C \epsilon^{1+1 / 4} / 2$, then for $0<t \leq \lambda \sin (\theta)-C \epsilon^{1 / 4}$ we get that $v_{t} \leq\left(1-C \epsilon^{1 / 4}\right) u_{2}$ on $\left\{x_{n}=C M \epsilon\right\} \cap Q_{1-C \epsilon}$, and hence $\bar{v}_{t}=v_{t}+\eta w \leq u_{2}$ there.

To show that $\bar{v}_{t} \leq u_{2}$ on $\partial Q_{1-C \epsilon} \cap\left\{\left|x_{n}\right| \leq C M \epsilon\right\}$ we notice that $\phi_{t}=1$ close to $\partial Q_{1-C \epsilon}$ since $\gamma=\sqrt{\epsilon} \gg \epsilon$. Therefore $v_{t} \leq\left(1-C \epsilon^{1 / 4}\right) u_{2}$ on $\partial Q_{1-C \epsilon} \cap\left\{\left|x_{n}\right| \leq\right.$ $C M \epsilon\}$. By the boundary Harnack inequality we also get $w \leq v_{t}$ on $\partial Q_{1-C \epsilon} \cap\left\{\left|x_{n}\right| \leq\right.$ $C M \epsilon\}$, and therefore $\bar{v}_{t} \leq u_{2}$ on that set. 
In particular, $\bar{v}_{t} \leq u_{2}$ on the boundary of $Q_{1-C \epsilon} \cap\left\{\left|x_{n}\right| \leq C M \epsilon\right\}$ for our choice of constants.

Now for $t=0$ we have $\bar{v}_{t}<u_{0}$ in $Q_{1-C \epsilon} \cap\left\{\left|x_{n}\right| \leq C M \epsilon\right\}$. Let us increase $t$ until the graph of $\bar{v}_{t}$ touches $u_{2}$ for the first time at $t=t_{0}$.

We claim that $t_{0}$ cannot be in the range of $t$ specified above. If $t_{0}$ was in the range specified, then $\bar{v}_{t} \leq u_{2}$ on the boundary of $Q_{1-C \epsilon} \cap\left\{\left|x_{n}\right| \leq C M \epsilon\right\}$, and since $L \bar{v}_{t} \geq 0$ away from the free boundary (by Lemma 6.6), we know that the free boundaries of $\bar{v}_{t_{0}}$ and $u_{2}$ must touch. However, by Lemma 6.9 we know that $\bar{v}_{t}$ is a sub-solution and from Lemma 6.7 all its free boundary points are regular; therefore by Lemma 6.4 this is not possible.

Theorem 6.12. Let $u$ be a solution to our free boundary problem satisfying $\| u-$ $x_{n} \|_{Q_{1}} \leq \delta / 2$, where $\delta \leq \delta_{0}$ for a small enough constant $\delta_{0}$. Then the free boundary is a Lipschitz graph in $Q_{3 / 4}$.

Proof. Pick a $\theta>2 \pi / 3$ and let $\epsilon$ be so small that

$$
\sum_{j=1}^{\infty}\left(C \epsilon \lambda^{j}\right)^{1 / 8} \leq \frac{1}{4}
$$

and

$$
\sum_{j=1}^{\infty}\left(C \epsilon \lambda^{j}\right)^{1 / 4} \leq \frac{\pi}{12}
$$

where $\lambda$ and the constants are from Proposition 6.11.

Then if

$$
\delta \leq \delta_{0}=\frac{\epsilon(1-\sin (\theta))}{2}
$$

we may iterate Proposition 6.11 and deduce that $u$ is fully monotone in the cone $\Gamma\left(7 \pi / 2, e_{n}\right)$ in the cube $Q_{3 / 4}$ and the theorem follows.

\section{LIPSCHITZ FREE BOUNDARIES ARE $C^{1, \alpha}$}

The idea of this section comes from [3, and we will rely heavily on the book [2] for proofs.

In a sense this section runs parallel with the previous one, and the general outline is very similar. Our starting point is that the solution $u$ is monotone for all directions in a certain cone $\Gamma\left(\theta, e_{n}\right)$ and the free boundary is Lipschitz. Then (Lemma 7.1 to Lemma 7.3) the function will actually be monotone in a larger cone away from the free boundary. This opens up for us to construct a similar subsolution as in the previous section, the difference being that the function $\phi$ can be chosen large in a ball away from the free boundary. With $\phi$ being large in a ball away from the free boundary, it can be chosen large in $B_{1 / 2}$ (see Lemma 7.4. in particular point (4)). This is enough, by the same methods as in the previous section, to show that $u$ will actually be monotone in a larger cone in $B_{1 / 2}$. Iterating this argument will imply $C^{1, \alpha}$ regularity of the free boundary.

Let us start with a Harnack inequality argument to show that $u$ is monotone in a larger cone away from the free boundary.

Lemma 7.1. Let $u$ be harmonic in $B_{1 / 4}$, monotone in $\Gamma\left(\theta, e_{n}\right), \sigma \in \Gamma\left(\theta, e_{n}\right)$ and $\nu=\frac{\nabla u(0)}{|\nabla u(0)|}$. Then there exists a small $\mu$ such that for any vector $\tau \in B_{\rho(\sigma)}(\sigma)$ 
where

we have $D_{\tau} u \geq 0$ in $B_{1 / 8}$.

$$
\rho(\sigma)=\mu \sigma \cdot \nu
$$

Proof. Take any $\sigma \in \Gamma\left(\theta, e_{n}\right)$. Then $D_{\sigma} u>0$ and

$$
\frac{D_{\sigma} u(0)}{D_{n} u(0)}=\frac{\sigma \cdot \nu}{\epsilon_{n} \cdot \nu} \geq \sigma \cdot \nu
$$

From the Harnack inequality it follows that

$$
\frac{D_{\sigma} u}{D_{n} u} \geq c_{0} \sigma \cdot \nu
$$

in $B_{1 / 8}$, that is, $D_{\sigma} u-c_{0}(\sigma \cdot \nu) D_{n} u \geq 0$. But $D_{n} u$ and $|\nabla u|$ are comparable, which means that $D_{\sigma} u-c_{1}(\sigma \cdot \nu)|\nabla u| \geq 0$ and the lemma follows.

The next lemma states that in the situation of the previous Lemma we actually get a strictly larger cone of monotonicity (for a proof see Theorem 4.2 in [2]).

Lemma 7.2. Let $0<\theta_{0}<\theta<\frac{\pi}{2}$, and for a unit vector $\nu$, let $H(\nu)=\{x ; x \cdot \nu=0\}$ be the hyperplane perpendicular to $\nu$. Assume that the cone $\Gamma\left(\theta, e_{n}\right) \subset\{x ; x \cdot \nu>0\}$.

Then there exists $\bar{\theta}$ and $\lambda=\lambda\left(\mu, \theta_{0}\right)<1$ such that

$$
\Gamma\left(\theta, e_{n}\right) \subset \Gamma(\bar{\theta}, \bar{e}) \subset \bigcup_{\sigma \in \Gamma\left(\theta, e_{n}\right)} B_{\rho(\sigma)}(\sigma),
$$

with $\rho(\sigma)=|\sigma| \mu \sigma \cdot \nu$ and

$$
\frac{\pi}{2}-\bar{\theta} \leq \lambda\left(\frac{\pi}{2}-\theta\right)
$$

Lemma 7.3. There exists a cone $\Gamma\left(\bar{\theta}_{1}, \overline{n u}{ }_{1}\right) \supset \Gamma\left(\theta, e_{n}\right)$ with $\bar{\delta}_{1} \leq \bar{\lambda} \delta_{0}$, where $\bar{\delta}_{1}=\frac{\pi}{2}-\bar{\theta}_{1}$ and $\bar{\lambda}=\bar{\lambda}\left(\theta_{0}, n\right)<1$. Also,

$$
D_{\sigma} u \geq 0
$$

in $Q_{1 / 8}\left(x_{0}\right)$ for every $\sigma \in \Gamma\left(\bar{\theta}_{1}, \bar{\nu}_{1}\right)$.

Proof. This is a simple consequence of the previous lemma and Lemma 7.1

As in the previous section we want to use comparison to deduce that $w(x)=$ $\sup _{y \in B_{\phi(x)}(x)} v(y) \leq u(x)$, where $v$ is a small translation of $u$. To improve the Lipschitz constant of the free boundary we want $\phi$ to be large in $B_{1 / 2}$. To use comparison we also need $w$ to be a sub-solution that is smaller than $u$ on the boundary.

If the free boundary is contained in a narrow strip around $x_{n}=0$ and $u$ is monotone in the cone $\Gamma\left(\theta, e_{n}\right)$, then, by Lemma $7.3, u$ is actually monotone in a larger cone in $B_{1 / 16}\left(\frac{3}{4} e_{n}\right)$. In particular, this means that we can choose $\phi$ somewhat large on $\partial B_{1 / 16}\left(\frac{3}{4} e_{n}\right)$ and still expect $w$ to be smaller than $u$ on $\partial B_{1 / 16}\left(\frac{3}{4} e_{n}\right)$. For $w$ to be a sub-solution $\phi$ needs to satisfy the equation in Lemma 6.5 The next lemma constructs such a $\phi$ that is also large in $B_{1 / 2}$.

Lemma 7.4. Let $0<r \leq \frac{1}{8}$. Then there exists $\lambda(r), h(r)>0$ a $C^{2}$ family of functions $\phi_{t}$ for $0<t<1$, defined in $\bar{B}_{1} \backslash B_{r / 2}\left(\frac{3}{4} e_{n}\right)$, such that

(1) $1 \leq \phi_{t} \leq 1+t h$,

(2) $\phi_{t} N \phi_{t} \geq C\left|\nabla \phi_{t}\right|^{2}$

(3) $\phi_{t}=1$ in $B_{1} \backslash B_{7 / 8}$ and 
(4) $\phi_{t} \geq 1+\lambda$ th in $B_{1 / 2}$,

(5) $\left|\nabla \phi_{t}\right| \leq C t h$.

Proof. Let $1 \leq \psi_{0} \leq 2$ be a smooth super-solution to $N \psi_{0}=0$ in $B_{1} \backslash B_{r / 2}\left(3 / 4 e_{n}\right)$, with boundary values $\psi_{0}=1$ on $\partial B_{r / 2}\left(3 / 4 e_{n}\right)$ and $\psi_{0}=2$ outside $B_{7 / 8}$. We may also take $\psi_{0} \leq 2-\gamma$ on $B_{1 / 2}$ for a $\gamma>0$.

We can now choose

$$
\phi_{t}=1+\operatorname{th} \frac{\psi_{0}^{1 /\left(1-c-2^{1 /(1-c)}\right)}}{1-2^{1 /(1-c)}}
$$

for an appropriately chosen $c$. For details see [13].

Parallel with the previous section we also need to make a small adjustment to our function to make it a sub-solution across the free boundary. This is done in the following lemma whose proof follows along the lines of Lemma 6.9 or of Lemma 4.12 in [2].

Lemma 7.5. Let $u$ be a solution to our free boundary problem and let $\phi_{t}$ be the family constructed in the previous lemma. Let $w_{t}$ be a continuous function in $\Sigma=$ $\bar{B}_{9 / 10} \backslash B_{1 / 8}\left(x_{0}\right)$ such that

$$
\begin{array}{ll}
\Delta w_{t}=0 & \text { in } \Omega^{+}\left(v_{\phi_{t}}\right) \cap \Sigma=\Omega_{t}, \\
w_{t}=0 & \text { in } \bar{\Sigma} \backslash \Omega_{t}, \\
w_{t}=0 & \text { on } \partial B_{9 / 10}, \\
w_{t}=u\left(x_{0}\right) & \text { on } \partial B_{1 / 8}\left(x_{0}\right) .
\end{array}
$$

Then for small constants $c, h$ and any $\epsilon>0$ small enough,

$$
\bar{v}_{t}=v_{\epsilon \phi_{t}}+c \epsilon w_{t}
$$

is a family of sub-solutions for $0<t<1$. Here $\bar{v}_{t}=\sup _{B_{\epsilon \phi_{t}(x)}} u$, with $\phi_{t}$ as in Lemma 7.4.

Now we are ready to put all the pieces together and get our gain in the cone of monotonicity.

Lemma 7.6. Let $u_{1} \leq u_{2}$ be two solutions of our free boundary problem in $B_{1}$, with the free boundary of $u_{2}$ being Lipschitz and containing the origin. Assume that in $B_{1-\epsilon}$

$$
v_{\epsilon}(x)=\sup _{B_{\epsilon}} u_{1} \leq u_{2},
$$

that for $b>0$, small,

$$
v_{\epsilon}\left(x_{0}\right) \leq(1-b \epsilon) u_{2}\left(x_{0}\right) \quad\left(x_{0}=\frac{3}{4} e_{n}\right)
$$

and that

$$
B_{1 / 8}\left(x_{0}\right) \subset \Omega^{+}\left(u_{1}\right) .
$$

Then, for $\epsilon$ small enough, there exists $\bar{\mu}>0$ such that

$$
v_{(1+\bar{\mu} b) \epsilon}(x) \leq u_{2}(x)
$$

in $B_{1 / 2}$. 
Proof. Let $\bar{v}_{t}=\sup _{B_{\epsilon \phi_{b t}(x)}} u_{1}+c b \epsilon w_{b t}$ be a family of sub-solutions, as in Lemma 7.5 Then $\bar{v}_{0}<u_{2}$ in $B_{9 / 10} \backslash \Omega^{+}\left(\bar{v}_{0}\right)$ by our assumption (7.11). Also, $\bar{v}_{0}<u_{2}$ in $\Omega^{+}\left(\bar{v}_{0}\right)$ by the Harnack inequality (Lemma 5.3) and the assumption that $w_{0}=u_{2}\left(x_{0}\right)$ on $\partial B_{1 / 8}\left(x_{0}\right)$. Here we might need to decrease $\epsilon$ by an arbitrarily small factor to get strict inequalities.

Now we slowly increase $t$ until the graph of $\bar{v}_{t}$ touches the graph of $u_{2}$ for the first time. Since $\bar{v}_{t}=\bar{v}_{0}<u_{2}$ on $\partial Q_{1}$ and $\bar{v}_{t}$ is a sub-solution wherever it is non-zero, the touching must happen either at the free boundary or on $\partial B_{1 / 8}\left(x_{0}\right)$. By Lemma 6.4 and an observation similar to (1) in Lemma 6.7 the touching cannot happen at the free boundary. Therefore the graph $\bar{v}_{t}$ will touch the graph of $u_{2}$ on $\partial B_{1 / 8}\left(x_{0}\right)$, so by Lemma $5.3 t \geq 1$ at the first touch.

In particular, $\bar{v}_{1} \leq u_{2}$ and the lemma follows by taking estimate (4) of Lemma 7.4 into consideration.

Applying the previous lemma on $u$ and a translation of $u$ gives an improvement of the Lipschitz constant.

Lemma 7.7. Let $u$ be a solution to our free boundary problem in $Q_{1}$ and assume that for some $0<\bar{\theta}<\theta \leq \frac{\pi}{2} u$ is monotonically increasing along any direction $\tau \in \Gamma\left(\theta, e_{n}\right)$. Then there exist $\lambda=\lambda(\bar{\theta}, n)<1$ and a cone $\Gamma\left(\theta_{1}, \nu_{2}\right) \supset \Gamma\left(\theta, e_{n}\right)$ such that

$$
\frac{\pi}{2}-\theta_{1}=\delta_{1} \leq \lambda \delta_{0}=\lambda\left(\frac{\pi}{2}-\theta_{0}\right),
$$

and in $Q_{1 / 2}$ we have $D_{\sigma} u \geq 0$ for every $\sigma \in \Gamma\left(\theta_{1}, \nu_{2}\right)$.

Proof. We apply Lemma 7.6 to $u_{1}(x)=u(x-\tau)$ and $u_{2}(x)=u(x)$. We only need to verify equation (7.11), which follows from Lemma 5.3 ,

Iterating this we may deduce the following theorem.

Theorem 7.8. Let $u$ be a solution to our free boundary problem in $Q_{1}$ with Lipschitz free-boundary. Then the free boundary is a $C^{1, \alpha}$ graph in $Q_{1 / 2}$.

Proof. If we iterate the previous lemma it follows that $u$ is monotonically increasing in $\Gamma\left(\theta_{j}, \nu_{j}\right)$ in $Q_{2^{-j}}(y)$ for every $y \in Q_{1 / 2}$ where $\pi / 2-\theta_{j} \leq \lambda^{j} \delta_{0}$. But this is exactly the same as $\operatorname{osc}_{Q_{r}(y)} \eta \leq C r^{\alpha}$ for $\alpha=-\ln _{2}(\delta)$, where $\eta$ is the normal of the free boundary.

\section{Proof of the Main theorem}

The proof of the main theorem is now easy. From Proposition 4.4 we know that for $\mu$-almost every $x^{0} \in \Gamma$ there is an $r>0$ such that

$$
\left|u\left(r x+x^{0}\right) /\left(\sup _{B_{r}\left(x^{0}\right)}|u|\right)-P\left(x_{n}\right)\right| \leq \delta \ll 1
$$

in $Q_{1}$ for a two plane solution $P$ with $\left\|P\left(x_{n}\right)\right\|_{L^{\infty}}\left(Q_{1}\right)=1$ (up to a rotation and possibly a normalization). Then from Theorem 6.12 it follows that the free boundary is a Lipschitz graph in $B_{r / 2}$ and from Theorem 7.8 we get that the free boundary is a $C^{1, \alpha}$ graph in $B_{r / 4}$.

So we only need to prove the $\sigma$-finiteness of the support to establish the theorem.

Lemma 8.1. The support of $\mu$ has $\sigma$-finite $(n-1)$-dimensional Hausdorff measure. 
Proof. It is enough to show that the set

$$
R=\left\{x \in \operatorname{spt}(\mu) ; \limsup _{r \rightarrow 0} \mu\left(B_{r}(x)\right) / r^{n-1}>0\right\}
$$

has full $\mu$ measure.

Once that is known, the lemma follows easily since

$$
\left\{x \in \operatorname{spt}(\mu) ; \limsup _{r \rightarrow 0} \mu\left(B_{r}(x)\right) / r^{n-1}>1 / j\right\}
$$

has finite $(n-1)$-dimensional Hausdorff measure for each $j$.

If $R$ does not have full measure, then the set $S=\operatorname{spt}(\mu) \backslash R$ carries some measure. In particular, $\mu$-almost every point $x \in S$ is a density point of $\mu$. Since $\mu$-almost every point in $\operatorname{spt}(\mu)$ has a neighborhood $B_{r}$, where the free boundary is a $C^{1, \alpha}$ graph, we should be able to find a density point $y \in S$ such that $\Gamma \cap B_{r}(y)$ is a $C^{1, \alpha}$ graph.

But then, by standard theory for the Harmonic measure, we have that $\mu$ is mutually absolutely continuous with respect to the Harmonic measure in $B_{r / 2}(y)$ and also that the Harmonic measure is mutually absolutely continuous with the surface measure of the free boundary. In particular, $\mu$ is mutually absolutely continuous with respect to the surface measure of the free boundary in $B_{r / 2}(y)$, which implies that

$$
\lim _{t \rightarrow 0} \frac{\mu\left(B_{t}(z)\right)}{t^{n-1}}>\delta
$$

for each $z \in \Gamma \cap B_{r / 2}(y)$, contradicting the fact that $y$ is a density point in $S$.

\section{REFERENCES}

1. Hans Wilhelm Alt, Luis A. Caffarelli, and Avner Friedman, Variational problems with two phases and their free boundaries, Trans. Amer. Math. Soc. 282 (1984), no. 2, 431-461. MR732100 (85h:49014)

2. Luis Caffarelli and Sandro Salsa, A geometric approach to free boundary problems, Graduate Studies in Mathematics, vol. 68, American Mathematical Society, Providence, RI, 2005. MR2145284 (2006k:35310)

3. Luis A. Caffarelli, A Harnack inequality approach to the regularity of free boundaries. I. Lipschitz free boundaries are $C^{1, \alpha}$, Rev. Mat. Iberoamericana 3 (1987), no. 2, 139-162. MR990856 (90d:35306)

4. — A Harnack inequality approach to the regularity of free boundaries. III. Existence theory, compactness, and dependence on X, Ann. Scuola Norm. Sup. Pisa Cl. Sci. (4) 15 (1988), no. 4, 583-602 (1989). MR.1029856 (91a:35170)

5. _ A Harnack inequality approach to the regularity of free boundaries. II. Flat free boundaries are Lipschitz, Comm. Pure Appl. Math. 42 (1989), no. 1, 55-78. MR973745 (90b:35246)

6. Luis A. Caffarelli, Lavi Karp, and Henrik Shahgholian, Regularity of a free boundary with application to the Pompeiu problem, Ann. of Math. (2) 151 (2000), no. 1, 269-292. MR 1745013 (2001a:35188)

7. Camillo De Lellis, Rectifiable sets, densities, and tangent measures, Zurich Lectures in Advanced Mathematics, European Math. Soc. (EMS), Zürich, 2008. vt127pp. MR2388959 $(2009 \mathrm{j}: 28001)$

8. Lawrence C. Evans, Classical solutions of fully nonlinear, convex, second-order elliptic equations, Comm. Pure Appl. Math. 35 (1982), no. 3, 333-363. MR649348 (83g:35038)

9. Mikhail Feldman, Regularity for nonisotropic two-phase problems with Lipschitz free boundaries, Differential Integral Equations 10 (1997), no. 6, 1171-1179. MR1608061 (99a:35277)

10. David Gilbarg and Neil S. Trudinger, Elliptic partial differential equations of second order, Classics in Mathematics, Springer-Verlag, Berlin, 2001, reprint of the 1998 edition. MR $1814364(2001 \mathrm{k}: 35004)$ 
11. Pertti Mattila, Geometry of sets and measures in Euclidean spaces, Cambridge Studies in Advanced Mathematics, vol. 44, Cambridge University Press, Cambridge, 1995, Fractals and rectifiability. MR 1333890 (96h:28006)

12. Pei-Yong Wang, Regularity of free boundaries of two-phase problems for fully nonlinear elliptic equations of second order. I. Lipschitz free boundaries are $C^{1, \alpha}$, Comm. Pure Appl. Math. 53 (2000), no. 7, 799-810. MR.1752439 (2001f:35448)

13. Regularity of free boundaries of two-phase problems for fully nonlinear elliptic equations of second order. II. Flat free boundaries are Lipschitz, Comm. Partial Differential Equations 27 (2002), no. 7-8, 1497-1514. MR1924475 (2003g:35232)

14. __ Existence of solutions of two-phase free boundary problems for fully nonlinear elliptic equations of second order, J. Geom. Anal. 13 (2003), no. 4, 715-738. MR2005161 (2005k:35438)

Mathematics Institute, University of Warwick, Coventry, CV4 7AL, United Kingdom

Department of Mathematical Sciences, Xi'an Jiaotong-Liverpool University, 111 Ren'ai Road, 215123 Suzhou (SIP), Jiangsu Province, People's Republic of China 